\title{
Effects of spatial curvature and anisotropy on the asymptotic regimes in Einstein-Gauss-Bonnet gravity
}

\author{
Sergey A. Pavluchenko ${ }^{1, a}$, Alexey Toporensky ${ }^{2,3}$ \\ ${ }^{1}$ Programa de Pós-Graduação em Física, Universidade Federal do Maranhão (UFMA), São Luís, Maranhão 65085-580, Brazil \\ ${ }^{2}$ Sternberg Astronomical Institute, Moscow State University, Moscow 119991, Russia \\ ${ }^{3}$ Kazan Federal University, Kazan 420008, Russia
}

Received: 2 November 2017 / Accepted: 29 April 2018 / Published online: 10 May 2018

(C) The Author(s) 2018

\begin{abstract}
In this paper we address two important issues which could affect reaching the exponential and Kasner asymptotes in Einstein-Gauss-Bonnet cosmologiesspatial curvature and anisotropy in both three- and extradimensional subspaces. In the first part of the paper we consider the cosmological evolution of spaces that are the product of two isotropic and spatially curved subspaces. It is demonstrated that the dynamics in $D=2$ (the number of extra dimensions) and $D \geq 3$ is different. It was already known that for the $\Lambda$-term case there is a regime with "stabilization" of extra dimensions, where the expansion rate of the three-dimensional subspace as well as the scale factor (the "size") associated with extra dimensions reaches a constant value. This regime is achieved if the curvature of the extra dimensions is negative. We demonstrate that it takes place only if the number of extra dimensions is $D \geq 3$. In the second part of the paper we study the influence of the initial anisotropy. Our study reveals that the transition from GaussBonnet Kasner regime to anisotropic exponential expansion (with three expanding and contracting extra dimensions) is stable with respect to breaking the symmetry within both three- and extra-dimensional subspaces. However, the details of the dynamics in $D=2$ and $D \geq 3$ are different. Combining the two described effects allows us to construct a scenario in $D \geq 3$, where isotropization of outer and inner subspaces is reached dynamically from rather general anisotropic initial conditions.
\end{abstract}

\section{Introduction}

Extra-dimensional theories had been known [1] even prior to General Relativity (GR) [2], but relatively well known they have become after work by Kaluza and Klein [3-5]. Since then the extra-dimensional theories evolved a lot but the main

a e-mail: sergey.pavluchenko@gmail.com motivation behind them remains the same-unification of interactions. Nowadays one of the promising candidate for a unified theory is $\mathrm{M} / \mathrm{string}$ theory.

The presence of the curvature-squared corrections in the Lagrangian of the gravitational counterpart of string theories is one of their distinguishing features. Scherk and Schwarz [6] demonstrated the need for the $R^{2}$ and $R_{\mu \nu} R^{\mu \nu}$ terms, while later Candelas et al. [7] proved the same for $R^{\mu \nu \lambda \rho} R_{\mu \nu \lambda \rho}$. Later it was demonstrated [8] that the only combination of quadratic terms that leads to a ghost-free nontrivial gravitation interaction is the Gauss-Bonnet (GB) term:

$L_{\mathrm{GB}}=L_{2}=R_{\mu \nu \lambda \rho} R^{\mu \nu \lambda \rho}-4 R_{\mu \nu} R^{\mu \nu}+R^{2}$.

This term, first found by Lanczos $[9,10]$ (therefore it is sometimes referred to as the Lanczos term) is an Euler topological invariant in (3+1)-dimensional space-time, but not in $(4+1)$ and higher dimensions. Zumino [11] extended Zwiebach's result on higher-than-squared curvature terms, supporting the idea that the low-energy limit of the unified theory might have a Lagrangian density as a sum of contributions of different powers of curvature. In this regard Einstein-Gauss-Bonnet (EGB) gravity could be seen as a subcase of the more general Lovelock gravity [12], but in the current paper we restrain ourselves to only quadratic corrections and so to the EGB case.

While considering extra-dimensional theories, regardless of the model, we need to explain where the additional dimensions are. Indeed, with our current level of experiments, we clearly sense three spatial dimensions and sense no presence of extra dimensions. The common explanation is that they are "compactified", meaning that they are so small that we cannot detect them. Perhaps, the simplest class of such theories are the theories with "spontaneous compactification". Exact solutions of this class have been known for a long time [13], but especially relevant for cosmology are those 
with a dynamical size of the extra dimensions (see [14-17] for different models). Notable recent studies include [18], where dynamical compactification of the $(5+1)$ Einstein-GaussBonnet model was considered; $[19,20]$, where different metric Ansätze for scale factors corresponding to $(3+1)$ - and extra-dimensional parts were studied, and [21-23], where we investigated general (e.g., without any Ansatz) scale factors and curved manifolds. Also, apart from cosmology, the recent analysis has focused on properties of black holes in Gauss-Bonnet [24-28] and Lovelock [29-33] gravities, features of gravitational collapse in these theories [34-36], general features of spherical-symmetric solutions [37], and many others.

When it comes to exact cosmological solutions, two most common Ansätze used for the scale factor are exponential and power law versions. Exponential solutions represent de Sitter asymptotic stages while power-law solutions represent Friedmann-like stages. Power-law solutions have been analyzed in $[14,38]$ and more recently in [39-43] so that by now there is an almost complete description of the solutions of this kind (see also [44] for comments regarding physical branches of the power-law solutions). One of the first considerations of the extra-dimensional exponential solutions was done by Ishihara [45]; later considerations included [46], as well as the models with both variable [47] and constant [48] volume; the general scheme for constructing solutions in EGB gravity was developed and generalized for general Lovelock gravity of any order and in any dimensions [49]. Also, the stability of the solutions was addressed in [50] (see also [51] for stability of general exponential solutions in EGB gravity), and it was demonstrated that only a handful of the solutions could be called "stable", while most of them are either unstable or have neutral/marginal stability.

If we want to find all possible regimes in EGB cosmology, we need to go beyond an exponential or power-law Ansatz and keep the scale factor generic. We are particularly interested in models that allow for dynamical compactification, so that we consider the spatial part as the warped product of three-dimensional and extra-dimensional parts. In that case the three-dimensional part is "our Universe" and we expect this part to expand, while the extra-dimensional part should be suppressed in size with respect to the threedimensional one. In [21] we demonstrated the existence of a regime when the curvature of the extra dimensions is negative and the Einstein-Gauss-Bonnet theory does not admit a maximally symmetric solution. In this case both the threedimensional Hubble parameter and the extra-dimensional scale factor asymptotically tend to constant values. In [22] we performed a detailed analysis of the cosmological dynamics in this model with generic couplings. Later in [23] we studied this model and demonstrated that, with an additional constraint on the couplings, Friedmann late-time dynamics in the three-dimensional part could be restored.
Recently, we have performed a full-scale investigation of the spatially flat cosmological models in EGB gravity with the spatial part being a warped product of three-dimensional and extra-dimensional parts [52-54]. In [52] we demonstrated that the vacuum model has two physically viable regimes - the first of them is the smooth transition from highenergy GB Kasner to low-energy GR Kasner. This regime appears for $\alpha>0$ at $D=1,2$ (the number of extra dimensions) and for $\alpha<0$ at $D \geq 2$ (so that at $D=2$ it appears for both signs of $\alpha$ ). The other viable regime is a smooth transition from high-energy GB Kasner to anisotropic exponential regime with expanding three-dimensional section ("our Universe") and contracting extra dimensions; this regime occurs only for $\alpha>0$ and at $D \geq 2$. In [53,54] we considered the $\Lambda$-term case and it appears that only a realistic regime is the transition from high-energy GB Kasner to anisotropic exponential regime; the low-energy GR Kasner is forbidden in the presence of the $\Lambda$-term so the corresponding transition does not occur. Also, if we consider joint constraints on $(\alpha, \Lambda)$ from our cosmological analysis and black holes properties, different aspects of AdS/CFT and related theories in the presence of the Gauss-Bonnet term (see [26,30,55-63]), the resulting bounds on $(\alpha, \Lambda)$ are (see [54] for details)

$$
\begin{aligned}
& \alpha>0, \quad D \geq 2, \quad \frac{3 D^{2}-7 D+6}{4 D(D-1)} \equiv \\
& \eta_{0} \geq \alpha \Lambda \geq \eta_{2} \equiv-\frac{(D+2)(D+3)\left(D^{2}+5 D+12\right)}{8\left(D^{2}+3 D+6\right)^{2}},
\end{aligned}
$$

where $\alpha$ is the Gauss-Bonnet coupling and $D$ is the number of extra dimensions.

The current paper is a natural continuation of our previous research on the properties of cosmological dynamics in EGB gravity. After a thorough investigation of spatially flat cases in [52-54], it is natural to consider spatially non-flat cases. Indeed, the spatial curvature affects inflation [64,65], so that it could change asymptotic regimes in other highenergy stages of the Universe evolution, and we are considering one of them. We already investigated the cases with negative curvature of the extra dimensions in [21-23], but to complete our description it is necessary to consider all possible cases. We are going to consider all possible curvature combinations to see their influence on the dynamics-we know the regime for the case with both subspaces being spatially flat and will see the change in the dynamics with the curvatures being non-flat. This allows us to find all possible asymptotic regimes in the spatially non-flat case; together with the results for the flat case, it will complete this topic.

Another important issue we are going to consider is the anisotropy within subspaces. Indeed, the analysis in [52-54] is performed under the conjecture that both three- and extradimensional subspaces are isotropic. The question is if the results are stable under small (or not very small) deviations 
of the isotropy of these subspaces. Finally, if we consider both effects, we could build a two-step scheme which allows us to qualitatively describe the dynamical compactification of an anisotropic curved space-time.

The structure of the manuscript is as follows: first we write down the equations of motion for the case under consideration. Next, we study the effects of curvature-we add all possible curvature combinations to all known existing flat regimes and describe the changes in the dynamics. After that we draw conclusions separately for the vacuum and the $\Lambda$ term regimes and describe their differences and generalities. After that we investigate the effects of anisotropy and find stability areas for different cases. Finally, we use both effects to build a two-step scheme which allow us to describe the dynamics of a wide class spatially curved models. In the end, we discuss the results obtained and draw the conclusions.

\section{Equations of motion}

Lovelock gravity [12] has the following structure: its Lagrangian is constructed from the terms

$L_{n}=\frac{1}{2^{n}} \delta_{j_{1} j_{2} \ldots j_{2 n}}^{i_{1} i_{2} \ldots i_{2 n}} R_{i_{1} i_{2}}^{j_{1} j_{2}} \ldots R_{i_{2 n-1} i_{2 n}}^{j_{2 n-1} j_{2 n}}$

where $\delta_{j_{1} j_{2} \ldots j_{2 n}}^{i_{1} i_{2} \ldots i_{2 n}}$ is the generalized Kronecker delta of the order $2 n$. One can verify that $L_{n}$ is Euler invariant in $D<$ $2 n$ spatial dimensions and so it would not give a nontrivial contribution to the equations of motion. Thus, the Lagrangian density for any given $D$ spatial dimensions is the sum of all Lovelock invariants (2), up to $n=\left[\frac{D}{2}\right]$, which give nontrivial contributions to the equations of motion:
$\mathcal{L}=\sqrt{-g} \sum_{n} c_{n} L_{n}$,

where $g$ is the determinant of metric tensor, $c_{n}$ is a coupling constant of the order of the Planck length in $2 n$ dimensions and summation over all $n$ in consideration is assumed.

The Ansatz for the metric is

$\mathrm{d} s^{2}=-\mathrm{d} t^{2}+a(t)^{2} \mathrm{~d} \Sigma_{(3)}^{2}+b(t)^{2} \mathrm{~d} \Sigma_{(\mathbf{D})}^{2}$,

where $\mathrm{d} \Sigma_{(3)}^{2}$ and $\mathrm{d} \Sigma_{(\mathbf{D})}^{2}$ stand for the metric of the two constant-curvature manifolds $\Sigma_{(3)}$ and $\Sigma_{(\mathbf{D})} \cdot{ }^{1}$ It is worth to point out that even a negative constant-curvature space can be compactified by making the quotient of the space by a freely acting discrete subgroup of $O(D, 1)$ [66].

The complete derivation of the equations of motion could be found in our previous papers, dedicated to the description of the particular regime which appears in this model [21,22]. It is convenient to use the following notation:

$A_{(1)}=\frac{\ddot{a}}{a}, \quad C=\frac{\dot{a} \dot{b}}{a b}, \quad B_{(1)}=\frac{\ddot{b}}{b}$,

$A_{(2)}=\frac{\left[\gamma_{(3)}+(\dot{a})^{2}\right]}{a^{2}}, \quad B_{(2)}=\frac{\left[\gamma_{(\mathbf{D})}+(\dot{b})^{2}\right]}{b^{2}}$,

and the following rescaling of the coupling constants:

$$
\begin{aligned}
& \alpha=\frac{(D+3)(D+2)(D+1)}{6} c_{0}, \\
& \beta=\frac{(D+1) D(D-1)}{6} c_{1}, \\
& \gamma=\frac{(D-1)(D-2)(D-3)}{6} c_{2} .
\end{aligned}
$$

Then the equations of motion could be written in the following form:

$$
\begin{aligned}
\mathcal{E}_{0}= & 0 \Leftrightarrow 0=\alpha+\beta\left(B_{(2)}+\frac{6}{D-1} C+\frac{6}{D(D-1)} A_{(2)}\right) \\
& +\gamma\left(B_{(2)}^{2}+\frac{12 A_{(2)} B_{(2)}}{(D-2)(D-3)}+\frac{24 C^{2}}{(D-2)(D-3)}+\frac{12 B_{(2)} C}{(D-3)}+\frac{24 A_{(2)} C}{(D-1)(D-2)(D-3)}\right) \\
\mathcal{E}_{i}= & \Leftrightarrow \Leftrightarrow 0=\alpha+\beta\left(B_{(2)}+\frac{4 A_{(1)}}{D(D-1)}+\frac{2 B_{(1)}}{D-1}+\frac{2 A_{(2)}}{D(D-1)}+\frac{4 C}{(D-1)}\right) \\
& +\gamma\left(B_{(2)}^{2}+\frac{16 A_{(1)} C}{(D-1)(D-2)(D-3)}+\frac{8 B_{(2)} C}{D-3}+\frac{8 A_{(1)} B_{(2)}}{(D-2)(D-3)}\right. \\
& \left.+\frac{8 A_{(2)} B_{(1)}}{(D-1)(D-2)(D-3)}+\frac{16 B_{(1)} C}{(D-2)(D-3)}+\frac{4 B_{(1)} B_{(2)}}{(D-3)}+\frac{4 A_{(2)} B_{(2)}}{(D-2)(D-3)}+\frac{8 C^{2}}{(D-2)(D-3)}\right)
\end{aligned}
$$

while the equation $\mathcal{E}_{a}=0$ reads

\footnotetext{
1 We consider the Ansatz for space-time in the form of a warped product $M_{4} \times b(t) M_{D}$, where $M_{4}$ is a Friedmann-Robertson-Walker manifold with scale factor $a(t)$, whereas $M_{D}$ is a $D$-dimensional Euclidean compact and constant-curvature manifold with scale factor $b(t)$.
} 


$$
\begin{aligned}
\mathcal{E}_{a}= & 0 \Leftrightarrow 0=\frac{D}{(D-4)} \alpha+\frac{(D-2)}{(D-4)} \beta\left(B_{(2)}+\frac{6 A_{(1)}}{(D-1)(D-2)}+\frac{2 B_{(1)}}{D-2}+\frac{6 A_{(2)}}{(D-1)(D-2)}+\frac{6 C}{(D-2)}\right) \\
& +\gamma\left(B_{(2)}^{2}+\frac{48 A_{(1)} C}{(D-2)(D-3)(D-4)}+\frac{12 B_{(2)} C}{D-4}+\frac{24 C^{2}}{(D-3)(D-4)}\right. \\
& +\frac{12 A_{(1)} B_{(2)}}{(D-3)(D-4)}+\frac{24 A_{(2)} B_{(1)}}{(D-2)(D-3)(D-4)}+\frac{24 B_{(1)} C}{(D-3)(D-4)}+\frac{4 B_{(1)} B_{(2)}}{(D-4)} \\
& \left.++\frac{12 A_{(2)} B_{(2)}}{(D-3)(D-4)}+\frac{24 A_{(2)} C}{(D-2)(D-3)(D-4)}+\frac{24 A_{(1)} A_{(2)}}{(D-1)(D-2)(D-3)(D-4)}\right) .
\end{aligned}
$$

\section{Influence of curvature}

In this section we investigate the impact of the spatial curvature on the cosmological regimes. As a "background" we use the results obtained in [52-54] — exact regimes for $\gamma_{(3)}=\gamma_{(\mathbf{D})} \equiv 0$ for both the vacuum and the $\Lambda$-term cases. As we use them as a "background" solutions, it is worth to quickly describe them all. All solutions found for both vacuum and $\Lambda$-term cases could be split into two groups-those with "standard" regimes as both past and future asymptotes and those with nonstandard singularity as one (or both) of the asymptotes. By the "standard" regimes we mean Kasner (generalized power-law) and exponential. In our study me encounter two different Kasner regimes: the "classical" GR Kasner regime (with $\sum p_{i}=\sum p_{i}^{2}=1$ where $p_{i}$ is the Kasner exponent from the definition of power-law behavior $\left.a_{i}(t)=t^{p_{i}}\right)$, which we denote $K_{1}\left(\right.$ as $\left.\sum p_{i}=1\right)$ and it is low-energy regime; and the GB Kasner regime (with $\sum p_{i}=3$ ), which we denote $K_{3}$ and it is high-energy regime. For a realistic cosmology we should have a highenergy regime as past asymptote and a low-energy as future asymptote, but our investigation demonstrates that potentially both $K_{1}$ and $K_{3}$ could play a role as past and future asymptotes [52]. Also we should note that $K_{1}$ exists only in the vacuum regime, while $K_{3}$ as past asymptote we encounter in both the vacuum and the $\Lambda$-term regimes (see [53] for details). The exponential regimes (where the scale factors depend upon time exponentially, so the Hubble parameters are constant) could be seen in both the vacuum and the $\Lambda$ term regimes and there are two of them - the isotropic and the anisotropic ones. The former of them corresponds to the case where all the directions are isotropized and, since we work in the multidimensional case, it does not fit the observations. On the contrary, the latter of them have different Hubble parameters for three- and extra-dimensional subspaces. For realistic compactification we demand expansion of the three- and contraction of the extra-dimensional spaces. The exponential solutions are denoted $E_{\text {iso }}$ for the isotropic and $E_{3+D}$ for the anisotropic case, where $D$ is the number of extra dimensions (so that, say, in $D=2$ the anisotropic exponential solution is denoted $E_{3+2}$ ).

The second large group are the regimes which have a nonstandard singularity as either of the asymptotes or even both of them. The nonstandard singularity is the situation which arises in nonlinear theories and in our particular case it corresponds to the point of the evolution where $\dot{H}$ (the derivative of the Hubble parameter) diverges at the final $H$; we denote it as $n S$. This kind of singularity is "weak" by Tipler's classification [67] and is type II in the classification by Kitaura and Wheeler $[68,69]$. Our previous research reveals that the nonstandard singularity is a wide-spread phenomenon in EGB cosmology, for instance, in the $(4+1)$-dimensional BianchiI vacuum case all the trajectories have $n S$ as either past or future asymptote [41]. Since a nonstandard singularity means the beginning or end of the dynamical evolution, either higher or lower values of $H$ are not reached and so the entire evolution from high to low energies cannot be restored; for this reason we disregard the trajectories with $n S$ in the present paper.

Thus, the viable (or realistic) regimes are limited to $K_{3} \rightarrow$ $K_{1}$ and $K_{3} \rightarrow E_{3+D}$ for the vacuum case and $K_{3} \rightarrow E_{3+D}$ for the $\Lambda$-term; we further investigate these regimes in the presence of curvature.

\subsection{Vacuum $K_{3} \rightarrow K_{1}$ transition with curvature}

First we want to investigate the influence of the curvature on the vacuum Kasner transition-the transition from Gauss-Bonnet Kasner regime $K_{3}$ to the standard GR Kasner $K_{1}$. We add curvature to either and both three- and extradimensional manifolds and see the changes in the regimes. We label the cases as $\left(\gamma_{3}, \gamma_{D}\right)$ where $\gamma_{3}$ is the spatial curvature of the three-dimensional manifold and $\gamma_{D}$-of the extradimensional. Therefore, for the $(0,0)$ case - the flat casewe have $K_{3} \rightarrow K_{1}$, as reported in [52]. Now if we introduce nonzero curvature, both $(1,0)$ and $(-1,0)$ do not change the regime and there remains $K_{3} \rightarrow K_{1}$. So we can conclude that $\gamma_{3}$ alone does not affect the dynamics. On the contrary, $\gamma_{D}$ 
does- $(0,1)$ has the transition changes to $K_{3} \rightarrow n S$, while $(0,-1)$ changes the transition to $K_{3} \rightarrow K_{D}$. This $K_{D}$ is a new but non-viable regime with $p_{3} \rightarrow 0$ and $p_{D} \rightarrow 1-\mathrm{a}$ regime with constant-size three dimensions and expanding as power-law extra dimensions, which makes the behavior in the expanding subspace Milne-like, caused by the negative curvature. Therefore, the curvature of the extra dimensions alone makes future asymptotes non-viable. Let us also note that for $D=2$ the original $K_{3} \rightarrow K_{1}$ regime exists for both $\alpha>0$ and $\alpha<0$, while for $D \geq 3$ it exists solely for $\alpha<0$. It appears that the $\alpha<0$ branch of the $D=2$ Kasner transition does not have $K_{3}^{D}$-instead, it has $n S$, so that $K_{3}^{D}$ exists only for $\alpha>0$ in $D=2$ and $\alpha<0$ in $D \geq 3$. If we include both curvatures, the situation changes as follows: for $(1,1)$ we have $K_{3} \rightarrow n S$; for $(1,-1)$ it is $K_{3} \rightarrow K_{3}^{D}$; for $(-1,1)$ it is $K_{3} \rightarrow n S$ and finally for $(-1,-1)$ it is $K_{3} \rightarrow K_{3}^{D}$.

The described regimes require some explanations. First of all, as we reported in [52], viable regimes have $p_{a}>0$ and $p_{D}<0$-indeed, we want expanding three-dimensional space and contracting extra dimensions to achieve compactification. Then it is clear why $\gamma_{3}$ alone does not change anything - with expanding scale factor, the effect of curvature vanishes. But most interesting is the effect of $\gamma_{D}=-1-$ indeed, negative curvature not just stops the contraction of the extra dimensions but starts their expansion, which changes the entire dynamics drastically. Now the extra-dimensional scale factor "dominates" and the three-dimensional one goes to a constant.

The scheme above has one interesting feature-as we described, $\gamma_{(\mathbf{D})}<0$ gives rise to regime with $p_{3} \rightarrow 0$ and $p_{D} \rightarrow 1$-but in $D=3$ this gives us a"would be" viable regime-indeed, if both subspaces are three-dimensional, as long as one is expanding and another is not, we could just call the expanding one "our Universe" and we have stabilized "extra dimensions". So that in $D=3$ there exists a regime with stabilized extra dimensions and a power-law expanding three-dimensional "our Universe". However, the viability of this regime needs more checks, and we leave this question to further study.

Thus the negative curvature of extra dimensions gives rise to new and an interesting regime $-K_{D}$ with expanding extra dimensions and constant-sized three-dimensional subspace. It is not presented in the spatially flat vacuum case, but it is also non-viable (except for $D=3$ ), so that it does not improve the chances for successful compactification. The only viable case is $K_{3} \rightarrow K_{1}$, which remains unchanged for $\gamma_{D}=0$.

\subsection{Vacuum $K_{3} \rightarrow E_{3+D}$ transition with curvature}

Now let us examine the effect of curvature on another viable vacuum regime- the transition from GB Kasner $K_{3}$ to the anisotropic exponential solution $E_{3+D}$. Similar to the previ- ously considered cases, for an anisotropic exponential solution to be considered as "viable", we require the expansion rate of the three-dimensional subspace to be positive and for the extra dimensions to be negative. Let us see what happens if we add a nonzero spatial curvature.

Similar to the previous case, the curvature of the threedimensional subspace $\gamma_{3}$ alone does not change the dynamics- $(1,0)$ and $(-1,0)$ both have the $K_{3} \rightarrow E_{3+D}$ regime. But unlike the previous case, the curvature of the extra dimensions $\gamma_{D}$ alone makes the future asymptotes singular-a power-law-type finite-time future singularity in the case of $\gamma_{D}=+1$ and nonstandard singularity in the case of $\gamma_{D}=-1$. The same situation remains in the cases that both subspaces have curvature - as long as $\gamma_{D} \neq 0$, the future asymptote is singular-either power-law or nonstandard, depending on the sign of the curvature.

Therefore, similar to the previous case, the only viable regime is unchanged, $K_{3} \rightarrow E_{3+D}$, which occurs if $\gamma_{D}=$ 0 . But unlike the previous case, this one does not give us interesting nonsingular regimes.

\section{$3.3 \Lambda$-term $K_{3} \rightarrow E_{3+D}$ transition with curvature}

Finally, let us describe the effect of curvature on the only viable $\Lambda$-term regime-the $K_{3} \rightarrow E_{3+D}$ transition described in $[53,54]$. The condition for viability is the same as in the described above cases-expansion of the threedimensional subspace and contraction of the extra dimensions. Our investigation suggests that the cases with $D=2$ and $D \geq 3$ are different; let us first describe the $D=2$ case. According to [53,54], there are three domains for the $\Lambda$-term case where the $K_{3} \rightarrow E_{3+D}$ transition take place.

We have: i) $\alpha>0, \Lambda>0, \alpha \Lambda \leq \zeta_{0}$ with $\zeta_{0}=1 / 2$ for $D=2,3$ and $\zeta_{0}=\left(3 D^{2}-7 D+6\right) /(4 D(D-1))$, ii) entire $\alpha>0, \Lambda<0$ domain and iii) $\alpha<0, \Lambda>0$, $\alpha \Lambda \leq-3 / 2$. Formally i) and ii) supplement each other to form a single domain $\alpha>0, \alpha \Lambda \leq \zeta_{0}$, but in i) there also exist isotropic exponential solutions, which, as we will see, affects the dynamics, so we consider these two domains separately. So for the domain of i), we have a regime unchanged if $\gamma_{(\mathbf{D})}=0$, isotropization $\left(K_{3} \rightarrow E_{\text {iso }}\right)$ if $\gamma_{(\mathbf{D})}<0$ and a nonstandard singularity $n S$ if $\gamma_{(\mathbf{D})}>0$. In the domain of ii), we again have unchanged $K_{3} \rightarrow E_{3+2}$ if $\gamma_{(\mathbf{D})}=0$ and $n S$ in all other (i.e. $\gamma_{(\mathbf{D})} \neq 0$ ) cases. Already here we can see the difference between i) and ii) domains. Finally, the domain of iii) has the same dynamics as ii). Therefore, in the domain where isotropic and anisotropic exponential solutions coexist, we have slightly richer dynamics, but neither of the regimes are viable; the only viable regime is unchanged $K_{3} \rightarrow E_{3+2}$ and it takes place if $\gamma_{(\mathbf{D})}=0$. Now if we consider the general $D \geq 3$ case, the resulting regimes are as follows: now the of i) and ii) have the same structureopposite to the $D=2$ case, the structure is as follows: the 
only viable regime is unchanged $K_{3} \rightarrow E_{3+D}$, which exists if $\gamma_{(\mathbf{D})}=0$; if $\gamma_{(\mathbf{D})} \neq 0$, we always have $n S$. The domain of iii) has the structure: unchanged $K_{3} \rightarrow E_{3+D}$ if $\gamma_{(\mathbf{D})}=0$, the "stabilization" (or "geometric frustration" regime [21,22]) if $\gamma_{(\mathbf{D})}<0$ and $n S$ if $\gamma_{(\mathbf{D})}>0$. This "stabilization" regime is the regime which naturally appears in the "geometric frustration" case and is described in $[21,22]$. In this regime the Hubble parameter, associated with three-dimensional subspace reaches a constant value while the Hubble parameter associated with the extra dimensions reaches zero (and so the corresponding scale factor-the "size" of the extra dimensions - reaches a constant value; the size of he extra dimensions "stabilizes").

Thus, in this last case-the $\Lambda$-term $K_{3} \rightarrow E_{3+D}$ transition-the "original" regime remains unchanged for $\gamma_{(\mathbf{D})}=0$. For nonzero curvature of extra dimensions, if it is positive, the future asymptote is singular, if it is negative, and $D \geq 3$, in the future we could have the regime with stabilization of extra dimensions, otherwise it is also singular.

We remind a reader that the geometric frustration proposal suggests that the dynamical compactification with stabilization of extra dimensions occurs only for those coupling constants in EGB gravity for which maximally symmetric solutions are absent. In turn, the absence of the maximally symmetric solutions means the absence of the isotropic exponential solutions, so that with negative curvature of the extra dimensions, isotropic and anisotropic exponential solutions cannot "coexist", which means that, for any set of couplings and parameters, only one of them could exist. The validity of this proposal has been checked numerically in $[53,54]$ for a larger number of extra dimensions, and now we see that it is valid also for the $D=3$ case.

It is not the same in the flat case-for instance, for $\alpha>0$, $\Lambda>0[53,54]$ we have both $K_{3} \rightarrow E_{\text {iso }}$ and $K_{3} \rightarrow E_{3+D}$ on different branches. If we turn on the negative curvature $\gamma_{(D)}<0$, the former of them remains, while the latter turns to $K_{3} \rightarrow n S$, a nonstandard singularity in $D=2$, or to the stabilization regime in $D>2$. This way we can see that $D=2$ is somehow pathological-in the presence of curvature, there are no realistic regimes in $D=2$ but they do exist in $D \geq 3$.

Finally, we made the same analysis starting from the exponential regime instead of the GB Kasner regime with the same number of expanding and contracting dimensions. The final fate of all trajectories appears to be the same. We will use this remark later in Sect. 5.

\subsection{Summary}

All three considered cases have the original regimes unchanged as long as $\gamma_{(\mathbf{D})}=0$. This means that the curvature of the three-dimensional world alone cannot change the future asymptote. For nonzero curvature of the extra dimensions, the situation is different in all three cases: in the vacuum $K_{3} \rightarrow E_{3+D}$ case all trajectories with $\gamma_{(\mathbf{D})} \neq 0$ are singular; in vacuum $K_{3} \rightarrow K_{1}$ we have a new regime but it is non-viable; finally, in $\Lambda$-term $K_{3} \rightarrow E_{3+D}$ case if $\gamma_{(\mathbf{D})}>0$ the future asymptote is singular, while for $\gamma_{(\mathbf{D})}<0$ there could be a viable regime with stabilization of the extra dimensions, but this regime occurs only when an isotropic exponential solution cannot exist and we have $D \geq 3$.

To conclude, it seems that the only important player in this case is the curvature of extra dimensions. And it is clear why is it so-from the requirements of viability we demand that the three-dimensional subspace should expand, while the extra dimensions should contract. The expansion of the three dimensions cannot be stopped by $\gamma_{(3)}>0$ nor by $\gamma_{(3)}<0$, which is why $\gamma_{(3)}$ does not influence the dynamics. On the other hand, extra dimensions are contracting, so both signs of extra-dimensional curvature affect it - a positive sign usually leads to a singularity (standard or not), while a negative sign could turn it to expansion (which is what we see in the $K_{D}$ regime).

\section{Influence of anisotropy}

In this section we address the problem of the anisotropy of each subspaces. In this case the equations of motion are different from (7)-(9); the metric Ansatz has the form

$g_{\mu \nu}=\operatorname{diag}\left\{-1, a_{1}^{2}(t), a_{2}^{2}(t), \ldots, a_{n}^{2}(t)\right\}$

substituting it into the Lagrangian and following the derivation described in Sect. 2 gives us the equations of motion:

$$
\begin{aligned}
& 2\left[\sum_{j \neq i}\left(\dot{H}_{j}+H_{j}^{2}\right)+\sum_{\{k>l\} \neq i} H_{k} H_{l}\right]+8 \alpha \\
& {\left[\sum_{j \neq i}\left(\dot{H}_{j}+H_{j}^{2}\right) \sum_{\{k>l\} \neq\{i, j\}} H_{k} H_{l}+3 \sum_{\{k>l>m>n\} \neq i} H_{k} H_{l} H_{m} H_{n}\right]-\Lambda=0}
\end{aligned}
$$

as the $i$ th dynamical equation. The first Lovelock term-the Einstein-Hilbert contribution-is in the first set of brackets and the second term-Gauss-Bonnet-is in the second set; $\alpha$ is the coupling constant for the Gauss-Bonnet contribution and we put the corresponding constant for the Einstein-Hilbert contribution to unity. Also, since in this section we consider spatially flat cosmological models, scale factors are not important in the physical sense and the equations are rewritten in terms of the Hubble parameters $H_{i}=\dot{a}_{i}(t) / a_{i}(t)$. Apart from the dynamical equations, we write down the constraint equation,

$$
2 \sum_{i>j} H_{i} H_{j}+24 \alpha \sum_{i>j>k>l} H_{i} H_{j} H_{k} H_{l}=\Lambda
$$


The relationship between $\left(c_{0}, c_{1}, c_{2}\right)$ and $(\alpha, \Lambda)$ is

$$
c_{0}=-\frac{6 \Lambda}{(D+3)(D+2)(D+1)} ; \quad c_{1}=\frac{6}{D+1} ; \quad c_{2}=6 D \alpha .
$$

First, let us consider $D=2$ case-it was demonstrated in [52-54] that the $D=2$ case has all regimes which higher-dimensional cases possess and does not have any extra regimes, so that the $D=2$ case is the simplest representative case. We seek an answer to the question-if the subspaces are not exactly isotropic (we consider the spatial part to be a product of three- and two-dimensional isotropic subspaces), how does it affect the dynamics? Is the asymptote still reached or not? Indeed, totally anisotropic (Bianchi-Itype) cosmologies are more generic, and if they still could lead to the asymptotes under consideration, this would widen the parameters and initial conditions spaces which could lead to viable compactification. Thorough investigation of $D=1$ case revealed [41] that only $n S$ is available as a future asymptote in the vacuum case (compare with [52] for regimes in $[3+1]$ spatial splitting), so that the problem of "losing" the regimes in the case of broken symmetry exists.

To investigate this effect, we solve the general equations (i.e., without $H_{1}=H_{2}=H_{3}=H$ and $H_{5}=\cdots=$ $H_{D-3}=h$ Ansatz implied) in the vicinity of the exact exponential and power-law solutions to see if the exact solution is reached in the course of the evolution, or if it is replaced with some other asymptote.

We start with vacuum regimes; according to [52], in the vacuum $D=2$ case at high enough $H_{0}$ (initial value for the Hubble parameter, associated with three-dimensional subspace), there are four combinations (two of them, $h_{1}$ and $h_{2}$ ) and $\alpha \lessgtr 0$. The first of the cases, $\alpha>0$ and $h_{1}$, gives the $K_{3} \rightarrow K_{1}$ transition. If we break the symmetries in both spaces, the stability of the regime is broken as well-in Fig. 1a we present the analysis of this case. There we present the regime depending on the initial conditions - we seek the regime change around the exact solution $H_{1}=H_{2}=H_{3}=$ 2.0, and $H_{4}=H_{5}=h_{0}$ is being found from the constraint equation (7); we fix $H_{3}=2.0$ and $H_{4}=h_{0}$ and change $H_{1}$ and $H_{2}$ and find $H_{5}$ from the constraint equation. The exact solution in question $\left(H_{1}=H_{2}=H_{3}=2.0, H_{4}=H_{5}=h_{0}\right)$ is depicted as a circle. The shaded area corresponds to the $K_{3} \rightarrow K_{1}$ regime, while the area which surrounds it corresponds to $K_{3} \rightarrow n S$. One can see that the stability region is quite small and any substantial deviation from the exact solution causes a nonstandard singularity. The second case, $\alpha>0$ and $h_{2}$, has the $K_{3} \rightarrow E_{3+2}$ regime. With broken symmetry the regime is conserved much better than the previous one-in Fig. 1b we present the analysis of this case. One can see that not just the area of the regime stability covers very large initial conditions, but this area is also unbounded. The typical evolution of such a transition is illustrated in Fig. 2a. The next case, $\alpha<0$ and $h_{1}$, has the $K_{3} \rightarrow K_{1}$ transition, just like the first one, and their stability is similar. Finally, the last case, $\alpha<0$ and $h_{2}$, governs the $K_{3} \rightarrow E_{\text {iso }}$ transition. If we break the symmetry for this case, the resulting stability area is quite similar to that of $K_{3} \rightarrow K_{1}$.

To summarize the results for the vacuum case, only $K_{3} \rightarrow$ $E_{3+2}$ - the transition from GB Kasner to anisotropic exponential solution-is stable. All other regimes-transitions to isotropic exponential solution and to GR Kasner-have much smaller stability areas and could be called "metastable". Formally, the basin of attraction of $K_{1}$ and isotropic expansion is nonzero and they are stable within it, but on the other hand its area is much smaller than that of $E_{3+2}$; so that comparing the two we decided to call $K_{3} \rightarrow E_{3+2}$ "stable", while $K_{3} \rightarrow K_{1}$ and $K_{3} \rightarrow E_{\text {iso }}$ are called "metastable".

Now let us consider the $\Lambda$-term case. According to [53], in the presence of the $\Lambda$-term the variety of the regimes is a bit different from the vacuum case. Again, there are two branches $\left(h_{1}\right.$ and $\left.h_{2}\right)$ and now in addition to variation in $\alpha$ there is variation in $\Lambda$ and in their product $\alpha \Lambda$.

The first case is $\alpha>0, \Lambda>0$. There on the $h_{1}$ branch we have $K_{3} \rightarrow E_{3+2}$ if $\alpha \Lambda \leq 1 / 2$ and $K_{3} \rightarrow n S$ if $\alpha \Lambda>1 / 2$. Another $\left(h_{2}\right)$ branch has $K_{3} \rightarrow E_{\text {iso }}$ regardless of $\alpha \Lambda$. All these three branches are stable-breaking the symmetry of both subspaces keeps the regimes as they are within wide vicinity of the exact solution, like in Fig. 1b. A stable solution $K_{3} \rightarrow E_{\text {iso }}$ as a future attractor for broken symmetry in both subspaces is illustrated in Fig. 2b. The next case to consider is $\alpha>0, \Lambda<0$; there the $h_{1}$ branch has $K_{3} \rightarrow E_{3+2}$, while $h_{2}$ has $K_{3} \rightarrow n S$ and the former of them is proved to be stable (the latter is not viable, so its stability is of little importance). Now let us turn to the $\alpha<0$ cases and the first one comes with $\Lambda>0$. At $\alpha \Lambda \geq-5 / 6$ both branches have the $K_{3} \rightarrow E_{\text {iso }}$ regime and both of them are metastableonly the initial conditions which are very close to the exact solution lead to $E_{\text {iso }}$, those beyond it lead to $n S$. On the contrary, at $\alpha \Lambda<-5 / 6$ on the $h_{1}$ branch we have $K_{3} \rightarrow$ $E_{3+2}$, while on the $h_{2}$ branch $K_{3} \rightarrow n S$ and again $E_{3+2}$ is stable. Finally, $\alpha<0, \Lambda<0$ has $K_{3} \rightarrow E_{\text {iso }}$ on $h_{1}$ and $K_{3} \rightarrow n S$ on $h_{2}$ and in this case $E_{\text {iso }}$ is stable.

In addition to the described above $D=2$ case, we also considered $D=3$. The methodology is the same and the results for the vacuum $K_{3} \rightarrow K_{1}$ are the same. But the results for both the vacuum and the $\Lambda$-term $K_{3} \rightarrow E_{3+3}$ transition are different. They are presented in Fig. 1c, where the initial conditions leading to $E_{3+3}$ are shaded with [3+3] added on them. One can see that the stability area is unbounded, as it was in the $D=2$ case, but there are differences as well. First, the upper part seems shrinked in comparison with $D=2$, so that starting from a vicinity of the exact solution, it is less probable to end up on $E_{3+3}$. Instead, we have $K_{3} \rightarrow E_{4+2}$-the exponential solution with four expand- 

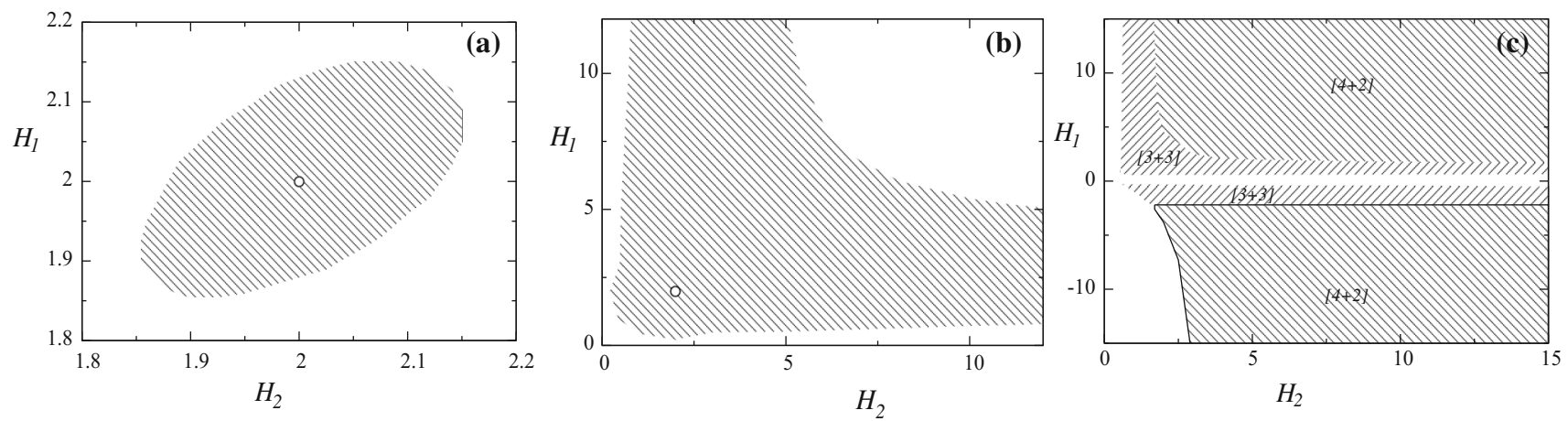

Fig. 1 Typical stability areas for vacuum $K_{3} \rightarrow K_{1}$ regime on $\mathbf{a}$; vacuum and $\Lambda$-term $K_{3} \rightarrow E_{3+2}$ regime on $\mathbf{b}$; vacuum and $\Lambda$-term $K_{3} \rightarrow E_{3+3}$ (and possibly higher number of extra dimensions as well) regime on c (see the text for more details)
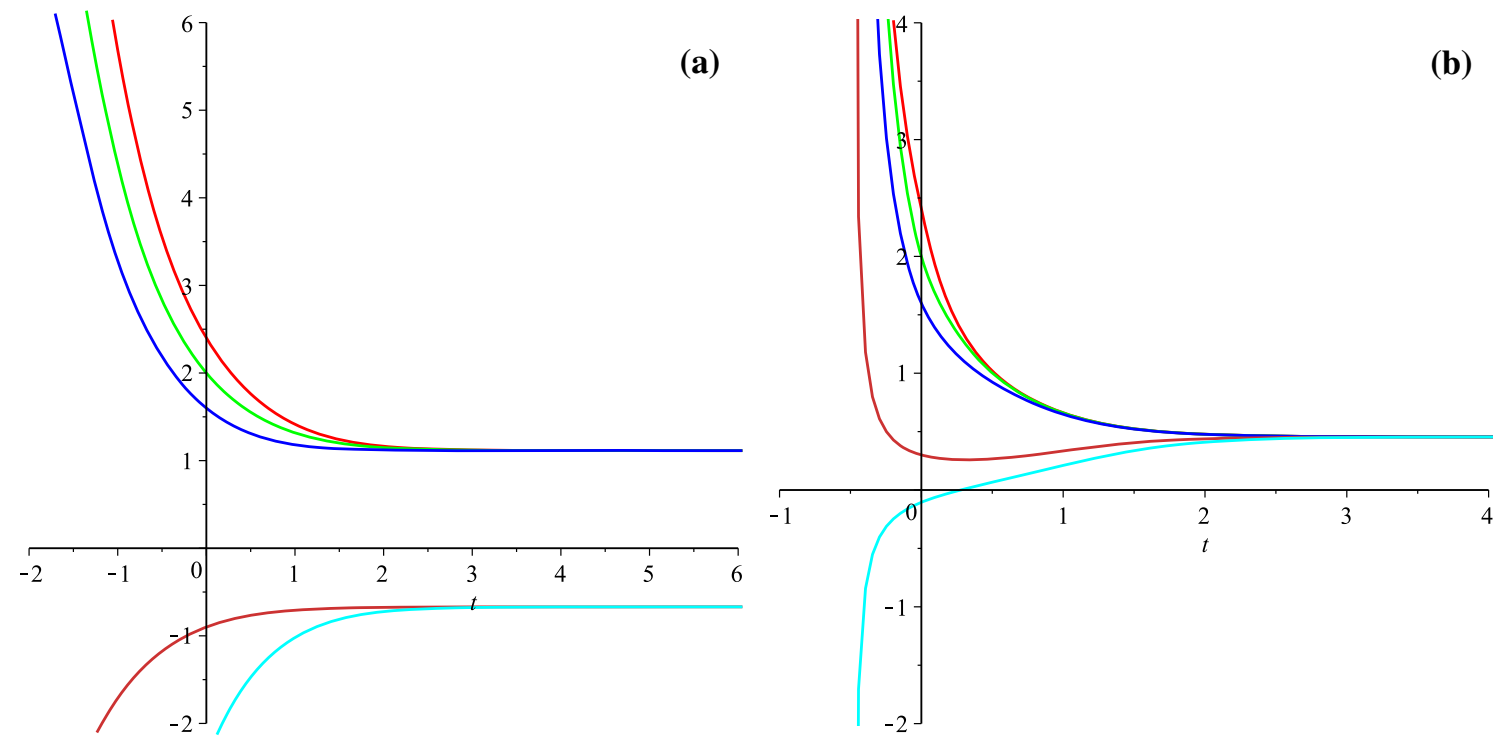

Fig. 2 Typical evolution curve for stable anisotropic a and isotropic $\mathbf{b}$ exponential solutions with broken symmetry in both subspaces in the $D=2$ case (see the text for more details)

ing and two contracting dimensions, which is, obviously, non-viable. In Fig. 3 we present $K_{3} \rightarrow E_{3+3}$ in panel (a) and $K_{3} \rightarrow E_{4+2}$ in panel (b), with the latter originating from some vast vicinity of the former. We also have some initial conditions starting from the negative values to lead to the exponential solution (which could "compensate" the loss in the upper part)—something we have never seen in $D=2$ - but this is the effect of the number of dimensionsin $D=2$, due to the smaller number of dimensions, the constraint is tighter, while in $D \geq 3$ it is more relaxed. The presence of the $E_{4+2}$ is also the effect of the higher number of extra dimensions-indeed, as we demonstrated in [47], in five spatial dimensions there is only one stable anisotropic exponential solution: $E_{3+2}$ (see $[50,51]$ for stability issues), while in six and higher there are more [49] and there is a chance to end up on another exponential solution. As the number of exact solutions grow with the number of dimen- sions, in higher dimensions it is probable to end up on another exponential solution, rather than $E_{3+D}$.

The black circle in Fig. 1c corresponds to the exact $E_{3+3}$ solution and one can see that the initial conditions are aligned along $H_{i}^{(0)} \sim H_{j}^{(0)}$. The same could be seen from the $D=2$ case as well (see Fig. 1b). The reason for it is quite clearindeed, with appropriate $H_{i}^{(0)}=H_{j}^{(0)}$ the exact $E_{3+D}$ solution is achieved explicitly, so that it is natural for the initial conditions to tend to this relation.

To conclude, we see that all $K_{3} \rightarrow E_{3+D}$ regimes in the $\Lambda$-term case are stable with respect to breaking the symmetry of both subspaces. On the other hand, another nonsingular regime, $K_{3} \rightarrow E_{\text {iso }}$, is stable only for $(\alpha>0, \Lambda>0)$ and $(\alpha<0, \Lambda<0)$. Finally, $K_{3} \rightarrow K_{1}$ in vacuum is also stable, but its basin of attraction is quite small and any substantial deviation from the exact solution destroys it. 

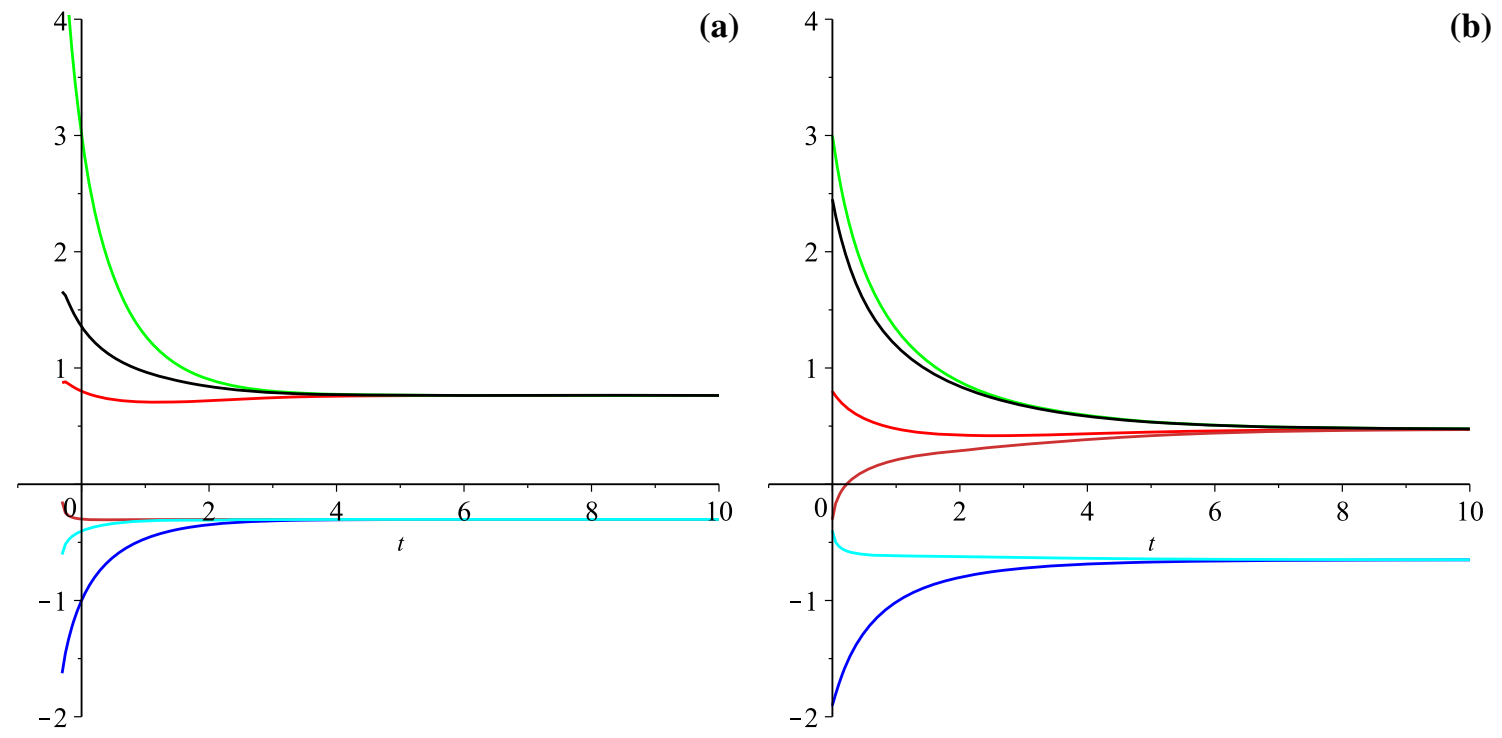

Fig. 3 Typical evolution curve for stable anisotropic [3+3] a and [4+2] b exponential solutions with broken symmetry in both subspaces in the $D=3$ case (see the text for more details)

\section{Two-step scheme for general spatially curved case}

The results of the two previous sections allow us to construct a scenario of compactification which satisfies two important requirements:

- the evolution starts from a rather general anisotropic initial conditions,

- the evolution ends in a state with three isotropic big expanding dimensions and stabilized isotropic extra dimensions.

The first part of the scenario in question uses the results of Sect. 4. We have seen there that on starting from a state in the dashed zone of Fig. 1b, c the flat anisotropic Universe tends to the exponential solution with three equal expanding dimensions. The initial conditions for such a behavior are not so restricted. From Fig. 1b we can see that the initial state should already have three expanding and two shrinking dimensions, however, since all Gauss-Bonnet Kasner solutions (as well as the usual GR Kasner solutions) should have at least one shrinking dimension, this requirement does not constrain possible initial states very seriously-in any cases we should expect that contracting dimensions are present in the initial conditions. Within this situation the dashed zone occupies a rather big part of the initial condition space of Fig. 1b, and any solution from this zone ends up in an exponential solution of the desired type.

In higher dimensions the situation is worsening, on the one hand — as it is seen from Fig. 1c, in $D \geq 3$, there is more than one stable anisotropic exponential solution, so that starting from the vicinity of exact $E_{3+3}$ solution we could end up in $E_{4+2}$ solution, which does not have realistic compactification. However, on the other hand, initial conditions with two expanding and four contracting dimensions can end up in $E_{3+3}$.

Suppose also that a negative spatial curvature is small enough at the beginning and starts to be important only after this transition to an exponential solution (which is established in the present paper only for a flat Universe) had already occurred. This condition allows us to glue the second part of the scenario, which requires negative spatial curvature of the inner space. We have seen in Sect. 3 that the exponential solution turns to the solution with stabilized extra dimensions in this case. As a result of these two stages a Universe starting from an initially anisotropic state with both outer expanding three-dimensional space and contracting inner space evolves naturally to the final stage with isotropic three big dimensions and isotropic and stabilized inner dimensions. The only additional condition for this scenario to be realized (in addition to starting from the appropriate zone in the initial conditions space) is that spatial curvature should become dynamically important only after the transition to an exponential solution occurs. As we mentioned in Sect. 3, this part (and so the entire scheme as well) works only for $D \geq 3$.

\section{Discussions and conclusions}

Prior to this paper, we completed a study of the most simple (but the most important as well) cases. The spatial part of these cases is the product of three- and extra-dimensional subspaces which are spatially flat and isotropic [52-54]. Thus, the obvious next step is consideration of these sub- 
spaces being non-flat and anisotropic, and that is what we have done in the present paper. Non-flatness is addressed by assuming that both subspaces have constant curvature, while anisotropy is addressed by breaking the symmetry between the spatial directions. The results of the curvature study suggest that the only viable regimes are those from the flat case with the requirement $\gamma_{(\mathbf{D})}=0$. Additionally, in the $\Lambda$-term case there is a "geometric frustration" regime, described in [21,22] and further investigated in [23] with the requirement $\gamma_{(\mathbf{D})}<0$.

Our study reveals that there is a difference between the cases with $\gamma_{(\mathbf{D})}=0$ and $\gamma_{(\mathbf{D})}<0$ : the former of them have only exponential solutions and the isotropic and anisotropic solutions coexist; the latter have the regime with stabilization of the extra dimensions (instead of a "pure" anisotropic exponential regime) and isotropic exponential regimes cannot coexist with regimes of stabilization - this difference was not noted before. The curvature effects also differ in cases with different $D$-in $D=2$ there is no stabilization of the extra dimensions, while in $D \geq 3$ there is.

In $D=3$ and $\gamma_{(\mathbf{D})}<0$ there is also an interesting regime in the vacuum case-the regime with stabilization of one and power-law expansion of the other three-dimensional subspaces; the viability of this regime for some compactification scenario needs further investigations.

The results of the anisotropy study reveal that the $K_{3} \rightarrow$ $E_{3+D}$ regime is always stable with respect to breaking the isotropy in both subspaces, meaning that within some vicinity of the exact $K_{3} \rightarrow E_{3+D}$ transition, all initial conditions still lead to this regime (see Fig. 2a). Although the area of the basin of attraction for this regime depends on the number of extra dimensions $D$, in $D=2$ it is quite vast (see Fig. 1b) and there are no other anisotropic exponential solutions, in $D=3$ (and higher number of extra dimensions) it seems smaller ${ }^{2}$ and there are initial conditions in the vicinity of $E_{3+D}$ which leads to other exponential solutions. In our particular example, $D=3$, presented in Fig. 1c, some of the initial conditions from the vicinity of $E_{3+3}$ end up in $E_{4+2}$ instead. We expect that in a higher number of extra dimensions the situation for $E_{3+D}$ would be more complicated and requires a special analysis.

Another viable regime, $K_{3} \rightarrow K_{1}$ from the vacuum case, as well as other non-viable regimes, are "metastable"formally they are stable, but their basin of attraction is much smaller compared to that of $E_{3+D}$ (see Fig. 1a).

Our study clearly demonstrates that the dynamics of the non-flat cosmologies could be different from flat cases and even some new regimes could emerge. In this paper we

\footnotetext{
2 To quantitatively address this question we need to introduce an appropriate measure and since the area is unbounded, this is not an easy task. Also, the answer will depends on the chosen measure, so we skip the quantitative analysis.
}

covered only the simplest case with constant-curvature subspaces leaving the most complicated cases aside-we are going to investigate some of them deeper in the papers to follow.

Now with both effects - the spatial curvature and anisotropy within both subspaces-being described, let us combine them. In the totally anisotropic case, as we demonstrated, a wide area of the initial conditions leads to anisotropic exponential solution (for the values of couplings and parameters when isotropic exponential solutions do not exist). Therefore, if we start from some vicinity of the exact exponential solution, and if the initial scale factors are large enough for the curvature effects to be small, we shall reach the anisotropic exponential solution with expanding three and contracting extra dimensions. After that the curvature effects in the expanding subspace are nullified, while in the contracting dimensions they are not. If it is the vacuum case, as we have shown earlier, as long as $\gamma_{(\mathbf{D})} \neq 0$ we encountered a nonstandard singularity, so that the vacuum case is pathological in this scenario. In the $\Lambda$-term case, as we reported earlier, for $\gamma_{(\mathbf{D})}=0$ we recover the same exponential regime, for $\gamma_{(\mathbf{D})}>0$ the behavior is singular and only for $\gamma_{(\mathbf{D})}<0$ we obtain the "geometric frustration" scenario $[21,22]$ with stabilization of the extra dimensions.

We can see that the proposed two-step scheme works only for the $\Lambda$-term case and only if $\gamma_{(\mathbf{D})}<0$-in all other cases it either provides trivial regimes, or it leads to singular behavior. Also, there is a minor problem with the number of extra dimensions - as we noted, the first stage of this schemereaching the exponential asymptote from initial anisotropyis best achieved in $D=2$ and the probability of reaching $E_{3+D}$ could decrease with the growth of $D$. On the other hand, the second stage- - when the negative curvature changes the contracting exponential solution for the extra dimensions into stabilization-is not present in $D=2$ and only manifest itself in $D \geq 3$. Thus, the described two-stage scheme works only in $D \geq 3$ and in this case the initial conditions for the first stage are already not so wide, though a fine-tuning of the initial conditions is not needed.

This finalize our paper: the analysis presented suggests that more in-depth investigations of both curvature and anisotropy effects are required-we have investigated and described the most simple but still very important cases-constant-curvature and flat anisotropic (Bianchi-Itype) geometries; in the papers to follow we are going to consider more complicated topologies.

Acknowledgements The work of A.T. is supported by RFBR grant 17-02-01008 and by the Russian Government Program of Competitive Growth of Kazan Federal University. The authors are grateful to Alex Giacomini (ICFM-UACh, Valdivia, Chile) for discussions.

Open Access This article is distributed under the terms of the Creative Commons Attribution 4.0 International License (http://creativecomm 
ons.org/licenses/by/4.0/), which permits unrestricted use, distribution, and reproduction in any medium, provided you give appropriate credit to the original author(s) and the source, provide a link to the Creative Commons license, and indicate if changes were made. Funded by SCOAP ${ }^{3}$.

\section{References}

1. G. Nordström, Phys. Z. 15, 504 (1914)

2. A. Einstein, Ann. Phys. (Berlin) 354, 769 (1916)

3. T. Kaluza, Sit. Preuss. Akad. Wiss. K1, 966 (1921)

4. O. Klein, Z. Phys. 37, 895 (1926)

5. O. Klein, Nature (London) 118, 516 (1926)

6. J. Scherk, J.H. Schwarz, Nucl. Phys. B 81, 118 (1974)

7. P. Candelas, G.T. Horowitz, A. Strominger, E. Witten, Nucl. Phys. B 258, 46 (1985)

8. B. Zwiebach, Phys. Lett. B 156, 315 (1985)

9. C. Lanczos, Z. Phys. 73, 147 (1932)

10. C. Lanczos, Ann. Math. 39, 842 (1938)

11. B. Zumino, Phys. Rep. 137, 109 (1986)

12. D. Lovelock, J. Math. Phys. (N.Y.) 12, 498 (1971)

13. F. Müller-Hoissen, Phys. Lett. B 163, 106 (1985)

14. N. Deruelle, L. Fariña-Busto, Phys. Rev. D 41, 3696 (1990)

15. F. Müller-Hoissen, Class. Quantum Gravity 3, 665 (1986)

16. J. Demaret, H. Caprasse, A. Moussiaux, P. Tombal, D. Papadopoulos, Phys. Rev. D 41, 1163 (1990)

17. G.A.M. Marugán, Phys. Rev. D 46, 4340 (1992)

18. E. Elizalde, A.N. Makarenko, V.V. Obukhov, K.E. Osetrin, A.E. Filippov, Phys. Lett. B 644, 1 (2007)

19. K.I. Maeda, N. Ohta, Phys. Rev. D 71, 063520 (2005)

20. K.I. Maeda, N. Ohta, JHEP 1406, 095 (2014)

21. F. Canfora, A. Giacomini, S.A. Pavluchenko, Phys. Rev. D 88, 064044 (2013)

22. F. Canfora, A. Giacomini, S.A. Pavluchenko, Gen. Relativ. Gravit. 46, 1805 (2014)

23. F. Canfora, A. Giacomini, S.A. Pavluchenko, A. Toporensky, Gravit Cosmol 24, 28 (2018). arXiv:1605.00041

24. D.G. Boulware, S. Deser, Phys. Rev. Lett. 55, 2656 (1985)

25. J.T. Wheeler, Nucl. Phys. B 268, 737 (1986)

26. R.G. Cai, Phys. Rev. D 65, 084014 (2002)

27. T. Torii, H. Maeda, Phys. Rev. D 71, 124002 (2005)

28. T. Torii, H. Maeda, Phys. Rev. D 72, 064007 (2005)

29. D.L. Wilshire, Phys. Lett. B 169, 36 (1986)

30. R.G. Cai, Phys. Lett. 582, 237 (2004)

31. J. Grain, A. Barrau, P. Kanti, Phys. Rev. D 72, 104016 (2005)

32. R.G. Cai, N. Ohta, Phys. Rev. D 74, 064001 (2006)

33. X.O. Camanho, J.D. Edelstein, Class. Quantum Gravity 30, 035009 (2013)
34. H. Maeda, Phys. Rev. D 73, 104004 (2006)

35. M. Nozawa, H. Maeda, Class. Quantum Gravity 23, 1779 (2006)

36. H. Maeda, Class. Quantum Gravity 23, 2155 (2006)

37. M.H. Dehghani, N. Farhangkhah, Phys. Rev. D 78, 064015 (2008)

38. N. Deruelle, Nucl. Phys. B 327, 253 (1989)

39. S.A. Pavluchenko, Phys. Rev. D 80, 107501 (2009)

40. S.A. Pavluchenko, A.V. Toporensky, Mod. Phys. Lett. A 24, 513 (2009)

41. S.A. Pavluchenko, Phys. Rev. D 82, 104021 (2010)

42. V. Ivashchuk, Int. J. Geom. Methods Mod. Phys. 07, 797 (2010). arXiv:0910.3426

43. I.V. Kirnos, A.N. Makarenko, S.A. Pavluchenko, A.V. Toporensky, Gen. Relativ. Gravit. 42, 2633 (2010)

44. S.A. Pavluchenko, A.V. Toporensky, Gravit Cosmol 20, 127 (2014). arXiv: 1212.1386

45. H. Ishihara, Phys. Lett. B 179, 217 (1986)

46. I.V. Kirnos, S.A. Pavluchenko, A.V. Toporensky, Gravit Cosmol 16, 274 (2010). arXiv: 1002.4488

47. D. Chirkov, S. Pavluchenko, A. Toporensky, Mod. Phys. Lett. A 29, 1450093 (2014). arXiv:1401.2962

48. D. Chirkov, S. Pavluchenko, A. Toporensky, Gen. Relativ. Gravit. 46, 1799 (2014). arXiv:1403.4625

49. D. Chirkov, S. Pavluchenko, A. Toporensky, Gen. Relativ. Gravit. 47, 137 (2015). arXiv: 1501.04360

50. S.A. Pavluchenko, Phys. Rev. D 92, 104017 (2015)

51. V.D. Ivashchuk, Eur. Phys. J. C 76, 431 (2016)

52. S.A. Pavluchenko, Phys. Rev. D 94, 024046 (2016)

53. S.A. Pavluchenko, Phys. Rev. D 94, 084019 (2016)

54. S.A. Pavluchenko, Eur. Phys. J. C 77, 503 (2017)

55. M. Brigante, H. Liu, R.C. Myers, S. Shenker, S. Yaida, Phys. Rev. D 77, 126006 (2008)

56. M. Brigante, H. Liu, R.C. Myers, S. Shenker, S. Yaida, Phys. Rev. Lett. 100, 191601 (2008)

57. A. Buchel, R.C. Myers, JHEP 0908, 016 (2008)

58. D.M. Hofman, Nucl. Phys. B 823, 174 (2009)

59. J. de Boer, M. Kulaxizi, A. Parnachev, JHEP 1003, 087 (2010)

60. X.O. Camanho, J.D. Edelstein, JHEP 1004, 007 (2010)

61. A. Buchel, J. Escobedo, R.C. Myers, M.F. Paulos, A. Sinha, M. Smolkin, JHEP 1003, 111 (2010)

62. X.-H. Ge, S.-J. Sin, JHEP 0905, 051 (2009)

63. R.G. Cai, Q. Guo, Phys. Rev. D 69, 104025 (2004)

64. S.A. Pavluchenko, Phys. Rev. D 67, 103518 (2003)

65. S.A. Pavluchenko, Phys. Rev. D 69, 021301 (2004)

66. J.A. Wolf, Spaces of constant curvature, 4th edn. (Publish or Perish, Wilmington, Delaware USA, 1984), p. 69

67. F.J. Tipler, Phys. Lett. A 64, 8 (1977)

68. T. Kitaura, J.T. Wheeler, Nucl. Phys. B 355, 250 (1991)

69. T. Kitaura, J.T. Wheeler, Phys. Rev. D 48, 667 (1993) 